\title{
Imaging magnetic excitations in confined magnetic structures
}

\author{
I Neudecker, F Hoffmann, G Woltersdorf and C H Back \\ Institut für Experimentelle und Angewandte Physik, Universität Regensburg, Universitätsstrasse 31, \\ 93040 Regensburg, Germany
}

Received 1 March 2008, in final form 19 April 2008

Published 1 August 2008

Online at stacks.iop.org/JPhysD/41/164010

\begin{abstract}
In this paper we revisit some particular aspects concerning the dynamic magnetization of thin $\mathrm{Ni}_{80} \mathrm{Fe}_{20}$ discs. In particular we address details of the excitation spectrum of $\mathrm{Ni}_{80} \mathrm{Fe}_{20}$ discs subjected to in-plane magnetic cw microwave fields. In this case imaging the dynamic magnetization allows us to estimate the internal magnetic field of the ferromagnetic element.
\end{abstract}

\section{Introduction}

In recent years the understanding of normal modes in confined magnetic structures has advanced considerably [1]. Since the first successful experiments aiming at imaging magnetization dynamics in micrometre sized ferromagnetic elements [2], significant progress has been made, in particular with respect to imaging the normal mode spectrum of simple magnetic elements [3-16]. Various experimental techniques have been used ranging from Brillouin light scattering experiments to synchrotron based imaging techniques and time resolved magneto-optic Kerr microscopy (TRMOKE). Among the studies concerning mode spectra of confined magnetic structures, the object studied most frequently is the flat disc shaped $\mathrm{Ni}_{80} \mathrm{Fe}_{20}$ platelet [17]. With an appropriate ratio of thickness (typically around $10-50 \mathrm{~nm}$ ) to diameter (typically $500 \mathrm{~nm}-10 \mu \mathrm{m}$ ) this element is in the flux closed state where the magnetization lies in the plane of the disc and circulates around its centre. At the centre of the element a vortex is formed, i.e. an area where the magnetization points out of the plane or into the plane of the disc (polarization of the vortex $p= \pm 1)$.

The magnetic vortex configuration of these simple elements supports two classes of dynamic excitations in the zero external magnetic field. When the vortex is displaced from the centre, e.g. by applying a short in-plane magnetic tipping field, it will spiral back into its original position. The direction of its motion (clockwise or counterclockwise) depends solely on the polarization of the vortex $p$ and the vortex motion is described well by the Thiele equation [18]. This so-called gyrotropic motion of the vortex core has been studied in numerous experimental and theoretical papers and is not discussed in this paper.
A second class of excitations is dominated by the shape of the elements and by the magneto-static energy. It has been shown experimentally as well as theoretically that the magneto-static energy leads to two types of normal modes that are reminiscent of the excitations of thin magnetic films $[11,15]$. In particular two types of excitations are observed. (i) When the wave vector of the dynamic magnetization points perpendicular to the static magnetization (which circulates around the disc) positive dispersion is observed and the observed modes are reminiscent of the Damon-Eshbach (DE) excitations of thin films. (ii) When the wave vector of the dynamic magnetization is parallel with the static magnetization negative dispersion is observed and the observed modes correspond to the magneto-static backward volume modes (MSBVs) of thin films. These two classes of modes and the corresponding dispersion relations have been successfully studied by employing TRMOKE.

In principle, the gyrotropic vortex motion modulates the boundary conditions for the magneto-static modes and leads to an interaction between gyrotropic and magnetometric modes [19]. This interaction leads to a splitting of the MSBVlike modes and is discussed in [20].

Here, we show data that are obtained by employing TRMOKE. In contrast to earlier experiments [11] we do not use pulsed excitation of the magnetization. Instead single frequency $\mathrm{cw}$ microwave excitation is used to study the dynamic modes of $\mathrm{Ni}_{80} \mathrm{Fe}_{20}$ discs. The measurement technique is thus a combination of ferromagnetic resonance (FMR) with TRMOKE and is called FMR-TRMOKE throughout this paper [21-23]. We first show normal modes of a $\mathrm{Ni}_{80} \mathrm{Fe}_{20}$ disc measured in a zero applied magnetic field for both perpendicular and in-plane excitation and then focus on the dynamic magnetization observed in in-plane magnetic fields 
(a)
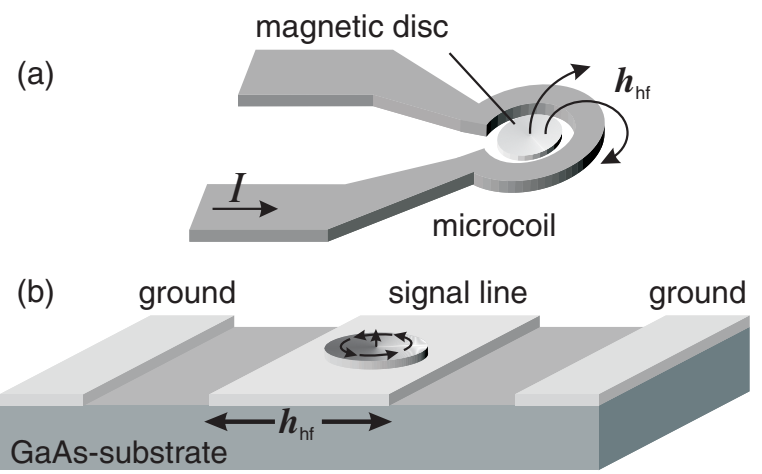

Figure 1. Experimental geometries: a $\mathrm{Ni}_{80} \mathrm{Fe}_{20}$ disc is placed (a) at the centre of a single turn micro-coil and $(b)$ on top of a coplanar strip line. In both cases a continuous wave microwave current is driven through the conductor which produces a microwave magnetic field $h_{\mathrm{hf}}$ at the position of the sample. In $(a)$ the microwave field points perpendicular to the plane of the disc. In $(b)$ the field is in the plane of the element. The magnetization of the ferromagnetic disc is in the flux closed vortex configuration in zero magnetic field.

large enough to expel the vortex from the disc. In this regime edge localized modes are visible. Detailed measurements allow us to estimate the internal magnetic fields of the samples.

\section{Experimental}

In our FMR-TRMOKE measurements a ferromagnetic sample is placed either inside a single turn micro-coil or on top of the central line of a coplanar strip line, see figure 1. In both cases a cw microwave current is applied to the high frequency wave guides which produces a magnetic microwave field $h_{\mathrm{hf}}$ at the position of the sample. In the case of a micro-coil the magnetic field points perpendicular to the plane of the disc, see figure $1(a)$ ). As the magnetization is in the plane of the disc (apart from the vortex in the centre which we neglect in the following), the $h_{\mathrm{hf}}$ field will predominantly excite radially symmetric modes (strictly this is only valid for perfectly symmetric magnetic elements and magnetic excitation fields). In the case of the coplanar waveguide, the $h_{\mathrm{hf}}$ field lies in the plane of the disc (see figure $1(b)$ ) which leads to the excitation of modes which always exhibit one nodal line in the azimuthal direction due to the excitation symmetry. The $h_{\mathrm{hf}}$ magnetic excitation field needs to be synchronized to the probing laser pulses. This is guaranteed by phase-locking the microwave generator which produces the $\mathrm{cw}$ rf current to our laser system, see figure 2. The spatial resolution of our Kerr setup is roughly $300 \mathrm{~nm}$. Details concerning this technique can be found in [21]. By measuring the TRMOKE signal as a function of the time delay between the microwave signal and the optical probe one obtains the amplitude and phase of the magnetic precession. In the following the phase is always chosen such that the dynamic modes are visible with maximum contrast. The experimentally detected modes are labelled according to their radial $(n)$ and azimuthal $(m)$ nodal lines. A mode with DE character possesses radial nodal lines; a mode with MBSV character has azimuthal nodal lines. Modes with mixed character are labelled according to their nodal lines $(n, m)$. Using cw excitation, normal modes $(n, 1)$ could indeed be

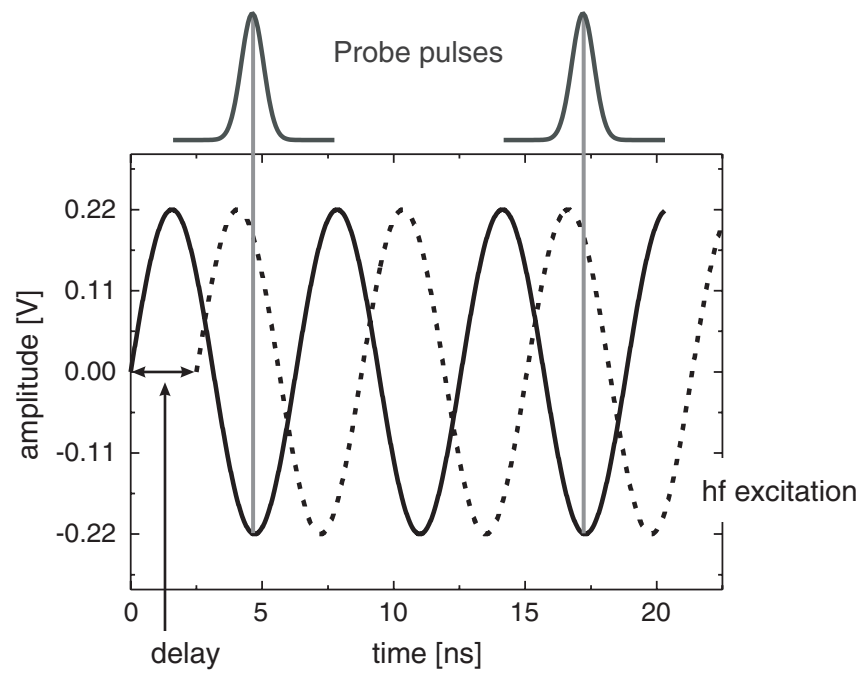

Figure 2. The correlation between the continuous wave excitation and the probe pulses for the resonant Kerr technique. The laser pulses arrive at a repetition rate of $80 \mathrm{MHz}$. Locking the cw microwave source to the clock of the laser ensures the laser pulses always arrive at the same phase of the excitation (shown for $160 \mathrm{MHz}$ cw excitation). A mechanical time delay between the source of the excitation and the sample allows one to shift the phase of the excitation with respect to the laser pulses. Thus, the response of the magnetic sample can be investigated at any phase of the exciting wave.

detected up to $n=5$. As an example, figure 3 shows the normal modes up to third radial order obtained from FMR-TRMOKE measurements for discs. We use a $3 \mu \mathrm{m}$ diameter disc $(15 \mathrm{~nm}$ thickness) for experiments using perpendicular excitation and a $4 \mu \mathrm{m}$ disc ( $20 \mathrm{~nm}$ thickness) for the in-plane experiments. The saturation magnetization is in both cases $\mu_{0} M_{\mathrm{S}}=1 \mathrm{~T}$. The resonance frequencies of the observed modes are in good agreement with earlier experiments using pulsed excitations and micromagnetic simulations (using the LLG code ${ }^{1}$ ), not shown. In the simulations we used both pulsed and cw excitations [24]. In figure 3(a) we show the measured polar Kerr contrast for the normal modes $(n, 0)$ up to $n=3$ accessible to out-of-plane excitation; figure $3(b)$ shows images of the polar Kerr contrast for in-plane excitation. In the latter case only modes with an azimuthal nodal line are observed. These additional nodal lines arise from the symmetry breaking due to the in-plane rf field. It is important to note that due to $\mathrm{cw}$ excitation the black/white contrast of the obtained images encodes an amplitude combined with the phase of the modes. In the experiment the phase is adjusted to obtain a maximum contrast. When comparing the experimental data with simulations, amplitude $A$ and phase $\varphi$ of the local Fourier transform data are combined by $A \sin \left(\varphi+\varphi_{0}\right)$ where $\varphi_{0}$ is an offset which is adjusted so that the data can be compared with the FMR-TRMOKE data, see, for example, figure 5.

The situation changes when an in-plane magnetic field is applied. In the following we show data obtained for a $4 \mu \mathrm{m}$ diameter $\mathrm{Ni}_{80} \mathrm{Fe}_{20}$ disc of $20 \mathrm{~nm}$ thickness which is subjected to a magnetic field in the plane of the disc strong enough to expel the vortex from the disc. In the convention used

1 LLG, the LLG code, can be found at http://llgmicro.home.mindspring.com/ 
(a)

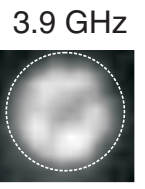

$(2,0)$
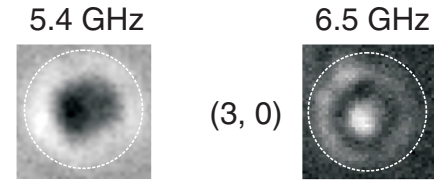

(b)

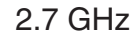

$(1,1)$

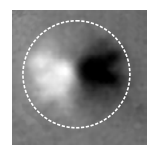

$(2,1)$

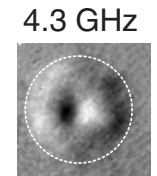

$5.4 \mathrm{GHz}$

$(3,1)$

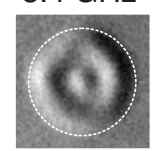

Figure 3. Normal modes observed using FMR-TRMOKE. (a) The first 'purely' radial modes $(n, 0)$ are visible for perpendicular excitation of a $3 \mu \mathrm{m}$ diameter $\mathrm{Ni}_{80} \mathrm{Fe}_{20}$ disc of $20 \mathrm{~nm}$ thickness. (b) The first modes $(n, 1)$ of a $4 \mu \mathrm{m}$ diameter $\mathrm{Ni}_{80} \mathrm{Fe}_{20}$ disc of $20 \mathrm{~nm}$ thickness as observed using in-plane excitation. Note that the excitation geometry breaks the symmetry and always leads to one azimuthal nodal line.

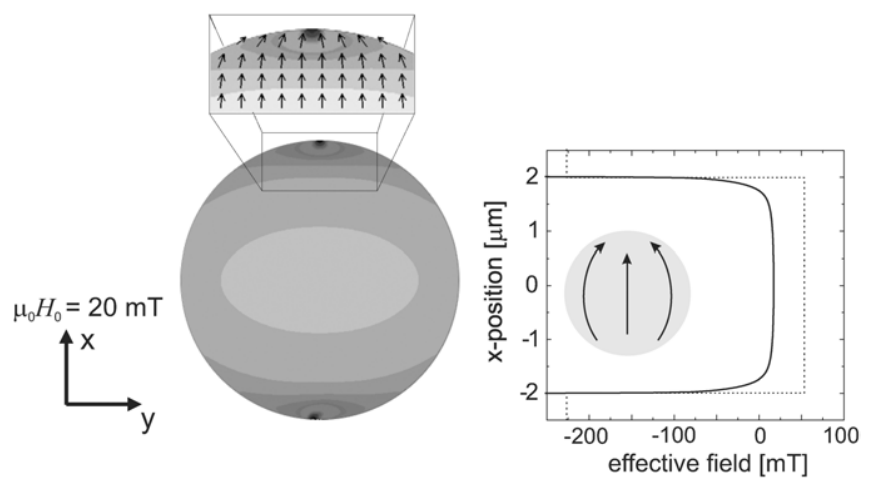

Figure 4. (a) The effective internal field of the $4 \mu \mathrm{m}$ diameter disc encoded by a black/white contrast at $\mu_{0} H_{0}=20 \mathrm{mT}$. In order to relate the static magnetization configuration $\boldsymbol{M}$ (represented by the arrows) to the effective field a small area of the edge region is enlarged. (b) A line scan along the external bias field reveals the field profile as a function of the position. The data were extracted from micromagnetic simulation.

here, the static magnetic field is applied along the $x$-direction, while the rf excitation field is applied along the $y$-direction. Due to the disappearance of the vortex core the element assumes a quasi-single domain state and the magnetization is now mostly pointing along the $x$-direction and assumes the so-called onion state with magnetic poles at the edges of the disc, see figure 4(a). The resulting stray field in turn locally softens the effective internal field of the system. This effect was first observed by Hiebert et al [2]. Figure 4 shows the internal effective field for the entire disc (figure 4(a)) and for a line scan across the disc centre parallel to the external field (figure $4(b)$ ) at an external field of $\mu_{0} H_{0}=20 \mathrm{mT}$. Due to the localized inhomogeneity of the internal field near the edges two regions inside the disc can be distinguished with respect to the effective field: a quasi-homogeneous region in the centre of the disc and a strongly inhomogeneous region in the vicinity of the disc edges. It is well known that the existence of the inhomogeneous internal field leads to so-called edge localized modes which exist in the soft regions while the central part of the element can be treated as a homogeneous region. This means that two types of modes can coexist in these micromagnetic elements. In fact, edge localized modes

have already been found previously in small stripe elements $[5,25,26]$ as well as in discs and in rings [27].

In figure 5 we show the dynamic magnetization recorded for a $\mathrm{Ni}_{80} \mathrm{Fe}_{20}$ disc of $20 \mathrm{~nm}$ thickness and a diameter of $4 \mu \mathrm{m}$ recorded at a frequency of $f=7.44 \mathrm{GHz}$ and as a comparison the result of a simulation performed at a slightly higher frequency of $f=8.1 \mathrm{GHz}$ in a magnetic field of $\mu_{0} H_{0}=20 \mathrm{mT}$. It is obvious from the images in figure 5 that the transverse quantization of the DE-like modes is observed not only in the centre region but also in the edge region. It is important to note that the simulation shown in figure 5 was carried out with the LLG code by applying a continuous wave excitation. When employing a short field pulse the mode localized at the disc edges is not observed clearly. The reason for this might be the interaction of the modes with other normal modes of the disc. Since with pulsed excitation, all modes are triggered simultaneously, the formation of the edge modes may be disturbed by the other modes.

When resonantly exciting the disc by means of a continuous wave hf field, one observes a standing wave located around the centre with a wave vector of $k_{\|}^{\text {centre }}=$ $2 \pi / \lambda=7.1 \times 10^{6} \mathrm{~m}^{-1}$ (see solid dots in figure 5). At the same frequency a standing wave is found near the edges of the disc (with respect to the direction of $\boldsymbol{M}$ ) with a wave vector of $k_{\|}^{\text {edge }}=8.2 \times 10^{6} \mathrm{~m}^{-1}$ (see open dots in figure 5). The corresponding internal field is shown by the black/white contrast in figure $4(a)$. Note that the wave vector does not change continuously with the increasing curvature of the disc boundary. Instead, two distinct regions-at the centre and the edge of the disc-with approximately constant wave numbers are found. These two regions correspond to the two different regions responsible for the localized edge modes observed parallel to $\boldsymbol{M}$ (see figure $4(b)$ ). Although the DE modes are quantized transverse to $\boldsymbol{M}$, the field parallel to the magnetization determines the corresponding wave numbers. In addition, despite the strong inhomogeneity found in the internal field the spatial distribution of the modes near the edges is rather uniform (figure 5). Even though the quantization of the edge modes could not be observed clearly in the simulation when applying pulsed excitation, the local Fourier transform of the time domain data reveals the quantized modes in the centre region. Consequently, their wave numbers could be extracted from a pulsed simulation and are shown in figure 6 (dots). Fitting the data points from the simulation to the dispersion relation of the DE mode

$$
\omega_{\mathrm{MSSW}}^{2}=\omega_{H}\left(\omega_{H}+\omega_{M}\right)+\frac{\omega_{M}^{2}}{4}\left(1-\mathrm{e}^{-2 k_{\|} t}\right)
$$

with the disc thickness as the only free parameter results in $t=20.5 \mathrm{~nm}$. The aspect ratio of the disc was accounted for by the effective demagnetizing factor $N_{\text {eff }}=0.97$ which reduces $\omega_{M}$. The fitted curve is represented by the solid line in figure 6 and shows excellent agreement with the simulations for data from both pulsed (dots) and continuous wave (square) excitation. The saturation magnetization and the effective internal field were determined by SQUID measurements and micromagnetic simulations, respectively $\left(\mu_{0} M_{\mathrm{S}}=1 \mathrm{~T}\right.$ and $\left.\mu_{0} H_{\text {eff }}=17 \mathrm{mT}\right)$. Using the same parameters and only 

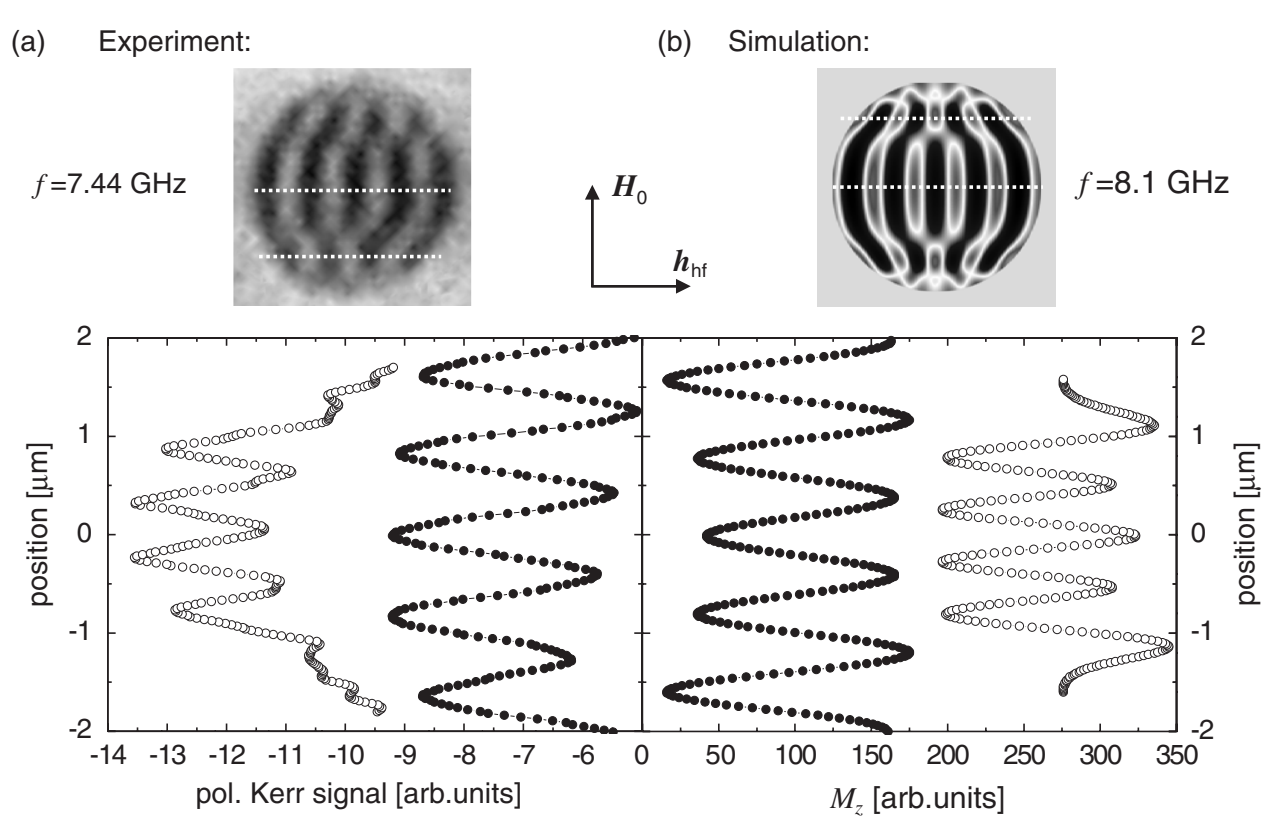

Figure 5. The DE-like mode obtained from (a) experiment at $f=7.44 \mathrm{GHz}$ and $(b)$ simulation at $f=8.1 \mathrm{GHz}$ in a magnetic field of $\mu_{0} H_{0}=20 \mathrm{mT}$. Line scans performed along the horizontal dotted lines indicated in the images show the dependence of the excitation wavelength on the position. The simulation was performed using a sinusoidal excitation.

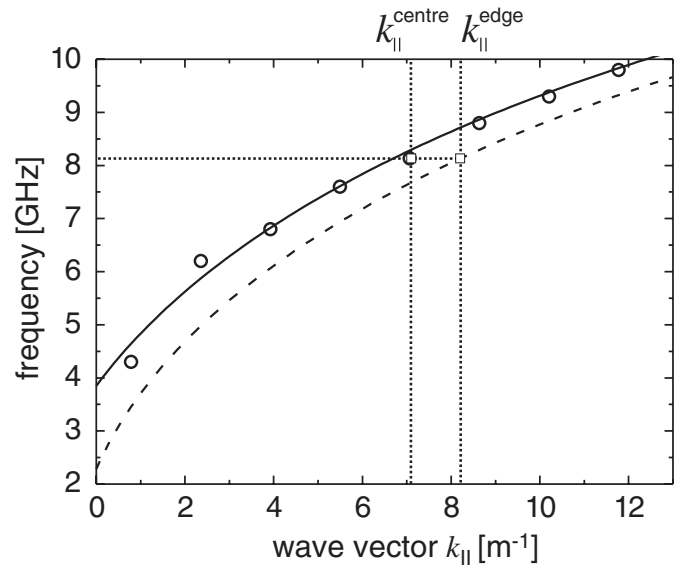

Figure 6. The frequencies of the DE modes extracted from simulations (symbols) and from the dispersion relation of the DE mode (lines). Data points from the simulations with continuous wave and pulsed excitations are represented by squares and dots, respectively. The theoretically derived dispersion relations are obtained by assuming different effective fields as described in the text. As a result different wave numbers $\left(k_{\|}^{\text {centre }}\right.$ and $\left.k_{\|}^{\text {edge }}\right)$ are observed in different regions at the same excitation frequency, indicated by the horizontal and vertical dotted lines.

assuming a weaker internal field $\left(\mu_{0} H_{\text {eff }}=6 \mathrm{mT}\right)$, one obtains a dispersion relation from equation (1), which traverses the data point obtained from the continuous wave excitation for the mode in the edge region (see the dashed line and square in figure 6). Indeed, due to the weaker effective field inside the edge region, the corresponding dispersion relation is shifted to lower frequencies with respect to the dispersion relation of the centre region. Therefore, the modes in the two regions have different wave numbers as observed in figure 5, even though they are excited at the same resonance frequency as illustrated by the dotted horizontal and vertical lines in figure 6 .
This effect might be employed in order to determine the effective internal field from measuring the wave vectors $k_{\|}$.

In summary we have shown that detailed investigations of dynamic magnetization using FMR-TRMOKE can lead to a detailed understanding of the micromagnetic objects. In particular, it is possible to extract internal magnetic fields from these measurements if other crucial parameters such as the film thickness and the effective saturation magnetization are known. It is, in principle, possible to extract these parameters from independent measurements with high accuracy.

\section{References}

[1] Hillebrands B and K O (ed) 2002-2006 Spin Dynamics in Confined Magnetic Structures I, II, III (Berlin: Springer)

[2] Hiebert W K, Stankiewicz A and Freeman M R 1997 Phys. Rev. Lett. 791134

[3] Acremann Y, Back C H, Buess M, Portmann O, Vaterlaus A, Pescia D and Melchior H 2000 Science 290492

[4] Choi B C, Belov M, Hiebert W K, Ballentine G E and Freeman M R 2001 Phys. Rev. Lett. 86708

[5] Park J P, Eames P, Engebretson D M, Berezovsky J and Crowell P A 2002 Phys. Rev. Lett. 89277201

[6] Tamaru S, Bain J A, van de Veerdonk R J M, Crawford T M, Covington M and Kryder M H 2002 J. Appl. Phys. 918034

[7] Park J P, Eames P, Engebretson D M, Berezovsky J and Crowell P A 2003 Phys. Rev. B 67 020403(R)

[8] Hiebert W K, Lagae L and Boeck J D 2003 Phys. Rev. B $68020402(\mathrm{R})$

[9] Barman A, Kruglyak V V, Hicken R, Kundrotaite A and Rahman M 2003 Appl. Phys. Lett. 823065

[10] Belov M, Liu Z, Sydora R D and Freeman M R 2004 Phys. Rev. B 69094414

[11] Buess M, Höllinger R, Haug T, Perzlmaier K, Krey U, Pescia D, Scheinfein M R, Weiss D and Back C H 2004 Phys. Rev. Lett. 93077207 
[12] Choe S-B, Acremann Y, Scholl A, Bauer A, Doran A, Stöhr J and Padmore H A 2004 Science 304420

[13] Demidov V E, Demokritov S O, Hillebrands B and Laufenberg M 2004 Appl. Phys. Lett. 852866

[14] Stoll H et al 2004 Appl. Phys. Lett. 843328

[15] Buess M, Knowles T P J, Höllinger R, Haug T, Krey U, Weiss D, Pescia D, Scheinfein M R and Back C H 2005 Phys. Rev. B 71104415

[16] Perzlmaier K, Buess M, Back C H, Demidov V E, Hillebrands B and Demokritov S O 2005 Phys. Rev. Lett. 94057202

[17] Kronmller H and Parkin S 2007 Handbook of Magnetism and Advanced Magnetic Materials (New York: Wiley)

[18] Thiele A A 1973 Phys. Rev. Lett. 30230

[19] Ivanov B A and Zaspel C E 2002 Appl. Phys. Lett. 811261

[20] Hoffmann F, Woltersdorf G, Perzlmaier K, Slavin A, Tiberkevich V, Bischof A, Weiss D and Back C 2007 Phys. Rev. B 76014416
[21] Neudecker I, Woltersdorf G, Heinrich B, Okuno T, Gubbiotti G and Back C 2006 J. Magn. Magn. Mater. 307148

[22] Woltersdorf G and Back C 2007 Phys. Rev. Lett. 99227207

[23] Woltersdorf G, Mosendz O, Heinrich B and Back C 2007 Phys. Rev. Lett. 99246603

[24] Neudecker I, Perzlmaier K, Hoffmann F, Woltersdorf G, Buess M and Back D W C 2006 Phys. Rev. B 73134426

[25] Jorzick J, Demokritov S O, Hillebrands B, Bailleul M, Fermon C, Guslienko K Y, Slavin A N, Berkov D V and Gorn N L 2002 Phys. Rev. Lett. 88047204

[26] Bayer C, Park J P, Wang H, Yan M, Campbell C E and Crowell P A 2004 Phys. Rev. B 69134401

[27] Neudecker I, Klui M, Perzlmaier K, Backes D, Heyderman L, Vaz C, Bland J, Rüdiger U and Back C 2006 Phys. Rev. Lett. 96057206 